\title{
Object Depots in the Genus Pogonomyrmex: Exploring the "Who," What, When, and Where
}

\author{
C. R. Smith ${ }^{1,3}$ and W. R. Tschinkel ${ }^{2}$ \\ Accepted March 16, 2005; revised August 8, 2005
}

\begin{abstract}
Harvester ants of the genus Pogonomyrmex collect and deposit many items on top of their nests. The depots of P. badius consist mostly of small charcoal fragments, while those of other species are primarily pebbles. Mature colonies can have hundreds of thousands of objects in their depot. In P. badius, the distributions of midden and charcoal about the mound are not completely overlapping, but are positively correlated in areas of overlap. Charcoal depots are isometric with colony size, but the amount of charcoal per colony size varies with season and site. The absence of the depot stimulates collection of nonfood objects. Recruitment to objects only occurs in the presence of food, and foragers choose objects based on size, but not color. An overview of current knowledge concerning depots in the genus indicates that depot formation is likely the ancestral state in the North American species. Furthermore, it is likely that selection is operating indirectly on these depots through selection on many dependent tasks.
\end{abstract}

KEY WORDS: pogonomyrmex; nest; depot; optimal foraging; indirect selection.

\section{INTRODUCTION}

Many animals forage for nonfood objects, and return to a central place where the objects are deposited or cached. For these central place foragers

\footnotetext{
${ }^{1}$ Program in Ecology and Evolutionary Biology, University of Illinois, 515 Morrill Hall, 505 S. Goodwin Avenue, Urbana, Illinois 61820.

${ }^{2}$ Department of Biological Science, Florida State University, Tallahassee, Florida 32306-4370.

${ }^{3}$ To whom correspondence should be addressed. E-mail: crsmith@life.uiuc.edu.
} 
the collection of objects at the nest provides a history of their foraging behavior that can illuminate the significance of the depot itself. For some animals, the evolutionary significance of the behavior is understood, such as foraging for nest materials in many birds. However, for the many others the significance is unknown (e.g., wood rats, woodpeckers, etc.). The collection of objects always comes at a price in time or energy, which may be high or low. Costs imply a benefit, but all too often benefits are ascribed without understanding the underlying behavioral mechanisms, and without testing whether a benefit exists and how it is manifested. Furthermore, an "adaptationist" approach (Gould and Lewontin, 1979) neglects the difference between the evolution (i.e., when it arises in time) and the maintenance of a behavior. Not distinguishing between these leaves open the possibility of misconstruing the meaning of the behavior for a species or group of related species. A second distinction that must be made when considering the adaptive nature of a depot is whether the depot itself or the behavior that causes the depot, foraging, is what is maintained by selection. Tinbergen's (1963) framework for understanding a behavior (ontogeny, causation, function and evolution) allows those assumptions to be satisfied.

Many ant species are described as depositing nonfood objects atop their nests (e.g., Aphaenogaster cockerelli, and Pheidole morrisi; personal observation), but none as extensively as harvester ants of the genus Pogonomyrmex. Many Pogonomyrmex species deposit large quantities of objects atop their nests, making their nests conspicuous landmarks (see Cole, 1968, Taber, 1998, and Smith, 2004 for photographs). These depots are typically located within the cleared areas around the nests, usually atop soil excavated from below. As particular species tend to collect objects of a uniform size these depots have been exploited by paleontologists searching for a particular size of fossil (Hatcher, 1896; Lull, 1915), geologists looking for particular rocks, archaeologists looking for artifacts (Reynolds, 1991), and indigenous tribes (at least historically) for religious purposes (Taber, 1998). Pogonomyrmex is a well-studied genus of ant (references in Taber, 1998; Gordon, 1999), and consequently there are many published observations of these object-depots, and several experimental studies.

Species differ in the composition of their depots, and their habitats. Therefore, it is often unknown whether species-specific behavior, or habitat limitations drive the size and composition of the depots. Moreover, it is often questioned whether the contents of the depots are derived from nest excavation or foraging. Although some species make modifications to the tops of their nests through excavation (Whitford, 2003), most objects are derived from foraging (Jorgensen and Porter, 1982; Reynolds, 1991, and personal observations). The proposed benefits conferred by the depots differ between species and include temperature modification (MacKay and 
MacKay, 1985), protection from erosion by water (Laundre, 1990) and wind (Whitford, 2003), and territory marking (Gordon, 1984). Few studies have examined the relationship between the depot and the ants, including how the depot is obtained, and how it is maintained.

As it is observed that multiple species within the genus have depots, how they are acquired becomes a central question if it is to be asserted that the behavior underlying the depots is the same across species (possibly homologous). Furthermore, observations on the types of objects collected across species can help in generalizing the behavior, and aid in teasing apart the confounding effects of object preference and availability. If it is determined that the behavior that gives rise to the depots (i.e., foraging for nonfood objects) is similar across species, the logical next step is to ask whether it is the behavior itself, or the depots themselves, that are important to the maintenance of the behavior. This distinction is important because selection may act on multiple levels (Sober and Wilson, 1998). If the depots themselves are merely a byproduct of foraging and do not benefit the colony, then the important question is not of the adaptive value of the depots, but how and why selection has shaped foraging behaviors.

This paper examines aspects of the evolution, ontogeny, and causation of object depot formation in the genus Pogonomyrmex, with a case study of the Florida harvester ant, $P$. badius. We describe the behavior in $P$. badius, including how depots are spatially arranged, and how depot size changes with colony size and season. We review the literature, poll researchers, and collect data on the types of objects found in depots and the rates at which they are collected in order to generalize the behavior across the genus. Furthermore, we investigate a stimulus for object collection in two species. In $P$. badius we assess several components of object choice in nonfood foraging, and examine the context-dependence of nonfood retrieval. A companion paper will address hypotheses regarding the function (i.e., adaptive significance) of the behavior for $P$. badius.

\section{MATERIALS AND METHODS}

\section{Ant and Site Descriptions}

Observations on P. badius were made at two sites in the Apalachicola National Forest (ANF), approximately $16 \mathrm{~km}$ from Tallahassee, Florida. Both sites are typical of $P$. badius habitat in that they have very sandy soil, a relatively deep water table ( $>1.5 \mathrm{~m}$ in most places), and abundant area with sparse shading. The two sites, Ant Heaven (AH) and Clear-cut (CC), are $3 \mathrm{~km}$ apart and differed in that the latter was clear-cut 4 years prior 
to our studies (1999), and hence had a completely open canopy. Due to the high intensity of disturbance generated by clear-cutting and absence of $P$. badius colonies in the forest surrounding the clear-cut it is likely that most colonies in this population are no more than 4-years old. The vegetation at AH consisted mainly of widely spaced longleaf pine (Pinus palustris), various oaks (Quercus sp.), and cactus (Opuntia sp.). At CC the vegetation was predominantly shiny blueberry (Vaccinium myrsinites), various small oaks and young $(<1 \mathrm{~m}$ tall) longleaf pine. $P$. badius nest density at $\mathrm{AH}$ was roughly twice that of $\mathrm{CC}, 5.2$ colonies per hectare vs. 2.8. Other dominant ant species at $\mathrm{AH}$, judging by nest and/or forager abundance, were Dorymyrmex bureni, Forelius pruinosus, Pheidole morrisi, Ph. dentata, Solenopsis carolinensis, S. geminata, and Trachymyrmex septentrionalis. At CC dominant ants were D. bureni, F. pruinosus, $S$. invicta, and $T$. septentrionalis.

Observations on P. barbatus were made at a site near Portal, Arizona in August 2003. The site was open with many cacti (Opuntia sp. and others) and mesquite (Prosopis sp.), and very rocky soil.

Pogonomyrmex nests are series of horizontal chambers and vertical shafts that can extend $>2 \mathrm{~m}$ into the earth (Tschinkel, 2004). The soil that is displaced by the ants to create their subterranean home is carried to the top of the nest and placed about the nest entrance, usually creating a volcano shape or mound. Mounds vary in height depending on the species in question. In P. badius the mound is typically only a few centimeters high, while in P. barbatus there is typically no visible mound. Atop this mound the ants place nonfood objects collected from the surrounding area. The sum of these pieces is the depot. The depots of $P$. $b a$ dius are predominantly made of small charcoal pieces, whereas in most western species, including $P$. barbatus, they are primarily constructed of pebbles.

\section{Depot Collection and Calculations}

Depots were collected to evaluate charcoal distribution about the nest, and depot size variation across colony size, sites, seasons and years. Entire $P$. badius charcoal depots were collected, dried (or water content was corrected for) and weighed. To estimate the percent of the mound covered by charcoal we measured both the covered and uncovered portions of the mound and subtracted them. For P. barbatus, nest area was measured, and a subsample $\left(400 \mathrm{~cm}^{2}\right)$ of the pebble covering was collected, dried and weighed in the lab. Subsamples were taken from an area equidistant from the nest entrance and the edge of the pebble covering, but random with 
respect to cardinal direction. To estimate the number of pieces of charcoal or pebbles in a depot we counted samples of known weight and extrapolated to the total weight of the depot.

Charcoal distribution on the nest was assessed by placing a $1-\mathrm{m}^{2}$ grid with divisions every $10 \mathrm{~cm}$ on a nest and collecting and weighing the charcoal inside each grid square $\left(100 \mathrm{~cm}^{2}\right)$. A contour map of charcoal density was generated by "blending" the charcoal weight per grid square using a distance-weighted least squares function with the tension set to zero (Statistica 6.0, Statsoft 2000). Charcoal was sieved to three sizes, and midden (trash items) using USA Standard Testing Sieves (W.S. Tyler Inc.) (large: $x>2.38 \mathrm{~mm}$, medium: $2<x<2.38 \mathrm{~mm}$, small: $1.76<x<2 \mathrm{~mm}$, and midden along with very small charcoal pieces: $0.85<x<1.76 \mathrm{~mm}$; sand passed through the 0.85 -mm sieve), weighed, and subsamples counted. This generated contour maps for all size classes of charcoal (by number and weight).

To analyze whether charcoal and midden are spatially associated about the nest, and whether their distributions are affected by the position of the nest entrance, we used Mantel tests. Two Mantel tests (code written by B. D. Inouye) were performed on data from each colony: between weights of charcoal and midden over the nest mound, and between the residuals of the previous analysis and the location of the nest entrance.

To test the relationship of colony size and depot size we used two procedures. The first is an indirect assessment that uses a proxy variable for colony size (mound area), and the second method directly estimates colony size by excavating whole colonies. To validate the use of mound size as a good proxy of colony size we directly measured this relationship. All analyses comparing sites or seasons were ANCOVAs, while allometry was tested using a standard regression model and a $t$-test for differences between slopes. Data in all of the analyses were log-transformed to stabilize variances.

Mound area as a proxy was assessed using data from 18 colonies at CC (May/June 2003) and 15 from AH (data from across four seasons in 1989). Mound area was tested as a predictor of colony size, and compared across sites.

Four datasets were used to examine the relationship between mound area and charcoal weight: data collected across seasons in 1989 from $\mathrm{AH}$ (using the colonies from Tschinkel, 1998, $n=22$ ), collections from AH in 2002 from May $(n=44)$ and then in October $(n=40)$, and a collection from CC in May $2003(n=17)$. Mound area was tested as a predictor of charcoal weight, and compared across datasets. A priori contrasts (from the ANCOVA model) were used to assess differences between sites within a season, seasons within a site, and years within a site (Table I). 
Table I. Comparison of Charcoal Depots Across Years, Seasons, and Sites

\begin{tabular}{lcl}
\hline \multicolumn{1}{c}{ Collection } & $\begin{array}{c}\text { Unadjusted } \\
\text { charcoal weight }(\mathrm{g})\end{array}$ & $\begin{array}{c}\text { Comparison (a priori) } \\
\text { contrasts from ANCOVA) }\end{array}$ \\
\hline AH-all seasons & 20 & $\square$ \\
AH-May 2002 & 100 & Year $^{\mathrm{a}} \square$ Season \\
AH-Oct. 2002 & 200 & Site $^{\mathrm{c}}$ \\
CC-May 2003 & 408 & \\
Average & 160 & \\
${ }^{a} F_{1,109}=110, p<0.01$. & & \\
${ }^{b} F_{1,109}=77, p<0.01$. & \\
${ }^{c} F_{1,109}=87, p<0.01$. &
\end{tabular}

We directly assessed the relationship between colony-size and charcoal weight with data from Tschinkel (1998), including previously unpublished data. Colony size was tested as a predictor of depot size.

\section{Estimation of Nonfood Collection Rate}

The absolute and relative nonfood collection rates were estimated to assess whether the removal of depots stimulated foraging for nonfood.

For P. badius the rate of charcoal collection was estimated using two methods. First, a forager trap (a plastic drinking cup buried until its edge was flush with the ground, flanked by two sheets of aluminum flashing) was placed approximately $1 \mathrm{~m}$ from the nest on the most active foraging trail. The number of ants and foraged items (insect, seed, charcoal and other) was counted and the rate was calculated as the number of pieces per minute. Traps were set up once and then covered when not in use. During each trial, the outbound entrance (i.e., nearest the colony) to the forager trap was blocked to ensure that only inbound foragers were trapped. Moreover, because the colonies were part of a longer-term experiment, all trapped foragers and their objects were placed on the mound once they had been counted. These traps were placed at 14 colonies. In seven colonies, the charcoal depot was removed and in the remaining seven it was removed and replaced. This method was used in two trials in May 2003 and is essentially a snapshot of ant activity, whereas method two is a longer-term cumulative measure, but estimated independently of the ants.

In method two, charcoal collection rate was estimated by collecting and weighing charcoal depots from the same colonies over successive dates. This method was used on 17 colonies (nine with depots removed and eight with depots removed and replaced) between the beginning of May and the date of colony collection (late May to early June). It required that we account 
for the amount of water in the charcoal that was weighed in the field. Because half of the colonies were weighed in the field and the other half both weighed in the field and then dried and reweighed in the lab, accurately correcting field weights for water content was possible. The difference in net charcoal gain by colonies in the two treatments was analyzed using an ANCOVA to account for variation in colony size (categorical factor $=$ treatment, continuous predictor $=\#$ dark workers, dependent variable $=$ net charcoal gain).

Estimating the collection rate of $P$. barbatus involved collecting returning foragers (with their load) for $10 \mathrm{~min}$. intervals. The number of collected ants and the identity of their loads were determined later in the lab. Observations were made in the late morning on the 20th of August 2003. Like method 1 for $P$. badius, this is a snapshot of forager activity, and was performed on colonies with depots removed $(n=4)$ and removed and replaced $(n=4)$.

The above experiments quantifying depot formation and stimulation lack an ideal third treatment group, unmanipulated colonies, due in part to the impossibility of quantifying the starting number of objects in the depot if it is not removed. Therefore, we confine our interpretation of these results to treatment differences, and use published data from other experiments to generalize our results.

\section{Choice and Context of Nonfood Foraging}

To examine whether object and food foraging were independent, we tested whether the collection of objects is dependent on presence or absence of food. As colonies are covered by a seemingly narrow size range of items of a uniform color, we tested whether colonies showed color and size preference for objects. Both food and objects used in these trials were novel, chopped toothpicks and mixed bird seed (mostly white millet). Novel objects were used over commonly collected items for two reasons: (1) foragers would not be biased by previous experience, and (2) foragers returning with experimental items could be distinguished from foragers retrieving objects away from the experimental area. All colonies in these trials were manipulated in the same way in order to facilitate observations. At each colony the direction of most foraging activity was determined by eye, and the ground cover (including vegetation) was removed from a $2 \times 1 \mathrm{~m}$ area in that direction to facilitate observations. Two sheets of metal flashing $\left(1 \mathrm{~m}\right.$ long $\times 15 \mathrm{~cm}$ high) placed at $\sim 45^{\circ}$ angles, facing out from the colony, approximately $0.5 \mathrm{~m}$ from the mound forced foragers into one trail and eased observations. Prior to any data collection, 
foragers were baited to the choice area with a small trail of seeds $(\sim 2 \mathrm{~g}$ of seed), thus assuring that colonies were exposed to choices in all trials.

To assess whether object retrieval differs depending on the context of presentation, we offered four colonies objects mixed with seeds and four only objects. Objects consisted of variable sizes of toothpick fragment (from $1 / 3$ to $1 / 25$ of a typical 7.5 -cm long toothpick) of varying color (red, green, blue, or yellow). All colors and sizes were in equal proportions in the offered pile. Equal proportions were determined by the exposed, two dimensional, surface area of fragments (directly proportional to weight in this case), which translates to the probability of discovery of the object. This experiment was done on 13 July, 2004. Two weeks later, the experiment was repeated with the same eight colonies, and treatments were reversed. Furthermore, in the second trial only red medium-sized objects were used. In both cases the presence of offered objects on top of the nest after $\sim 200 \mathrm{~min}$ was used as the response variable (the color and size of pieces was not documented). Objects were never collected when not offered with seeds. To test whether colonies offered objects with seeds were more likely to collect those objects, a binomial test was used on pooled data from the above two experiments (a total of eight colonies).

To test for color and size preference of foraged objects, four colonies (different from the eight above) were again offered toothpick pieces. Colonies were offered four colors of wood toothpicks (red, yellow, green and blue), in two size classes (large $=1 / 3$ toothpick and medium $=1 / 6$ ). They were offered $4 \mathrm{~g}$ of each color medium-size ( $\sim 100$ pieces) toothpick and $2 \mathrm{~g}$ of each color of large toothpick ( $\sim 50$ pieces), again equalizing the exposed surface area of each size class. We mixed $14 \mathrm{~g}$ of birdseed with the objects to ensure recruitment to piles. Colonies were offered object-seed piles on 14 July, 2004 and objects left in the piles and on the nest were collected on 16 July. Collected objects were sorted to color and size and counted. Ratios of object colors and sizes in the pile after 2 days were compared to the original ratios to determine whether piles became depleted in size or color (which would bias forager exposure to any class of objects). A nested ANOVA was used to analyze these data, where each replicate was the number of pieces collected (square root transformed to normalize variance) by a single colony, and the effects were object size, and color nested within size. Additionally, observations on the behaviors of workers retrieving objects, and the fate of those objects, were made.

Observations on species of Pogonomyrmex other than P. badius were made by the authors on trips through the southwestern United States and Mexico, obtained from other researchers in the form of personal communications, and from the literature. 


\section{RESULTS}

\section{P. badius}

Charcoal, on average, covered $82 \%( \pm 14 \%, n=40)$ of the mound area, leaving a circular area around the entrance uncovered. This pattern may be due, in part, to the continuous excavation of new chambers and placement of excavated soil in the area directly outside of the entrance (personal observation).

Foragers that return to the nest with a piece of charcoal typically take it into the nest, although they sometimes deposit it directly on the depot. Charcoal in the depot is arranged and rearranged throughout a daily cycle (Gordon, 1984), yet the spatial arrangement of charcoal was similar across colonies, and all size classes of charcoal. Midden such as discarded seed husks, on the other hand, was most often placed in a discrete pile located within the mound area. The discrete nature of the midden pile relative to the charcoal (Fig. 1) indicates that the ants are not treating charcoal as midden. However, in all three colonies tested, charcoal and midden were spatially correlated, where there was more trash there was more charcoal $(p<0.05)$. The deviation of the charcoal distribution from the trash distribution was random with respect to the nest entrance $(p>0.05)$.

The average weight of charcoal on 84 colonies of a range of sizes at $\mathrm{AH}$ in May and October was $228 \mathrm{~g}$ or 34,000 pieces, with the largest colonies having over $500 \mathrm{~g}$ and the smallest $5 \mathrm{~g}$, or approximately 77,000 and 660 pieces respectively. These estimates are conservative because they derive from the predictive regression for only the largest pieces $\left(\log _{10} \#\right.$ pieces $=$ $2.0927+1.0341 \times \log _{10}$ weight $\left.(\mathrm{g})\right)$.

The area of the mound provides a good proxy measure of colony size. Mound area explains $84 \%$ of the variance in colony size (number of mature workers) at $\mathrm{AH}$ and $59 \%$ at CC (Fig. 2). For a given mound area, colonies at AH were larger than those at CC $\left(F_{1,30}=29, p<0.001\right)$, but the slopes of the relationships between mound area and colony size for AH and CC were not significantly different from each other, 1.18 and 1.22 respectively, and neither was different than $1(t=1.27$ and $0.85, d f=13$ and 16 , and $p>0.2$ and 0.4 respectively). The mound area is isometric with the number of ants in a nest. Therefore, the number of workers in the nest is simply a multiple of the mound area, but this relationship differs between population/sites.

The amount of charcoal on a colony varied with season, site, and year (after adjusting for mound area). The slopes of the relationships between mound area and charcoal weight did not differ between the datasets (Fig. 3), and ranged from 0.7 to 0.87 . Moreover, all of these relationships are isometric (slopes are not different than $1, t<1.6$ and $p>0.1$ in all cases). 
a)

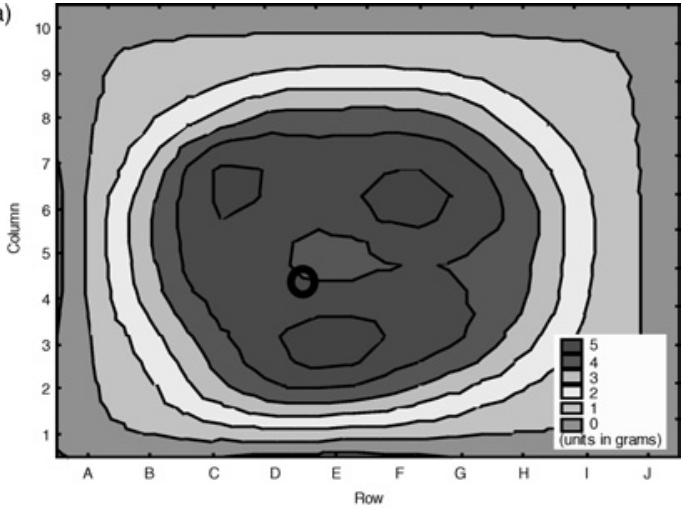

b)

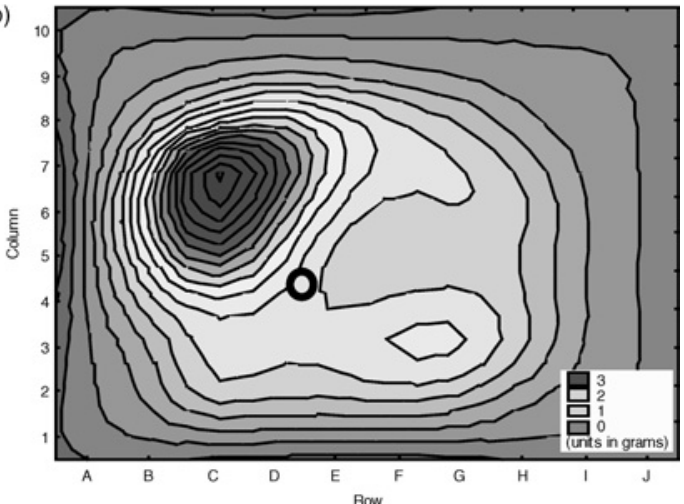

c)

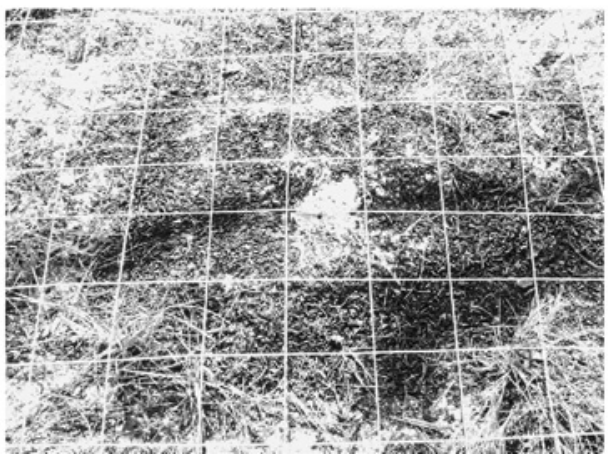

Fig. 1. Charcoal (a) and midden (b) distribution, an example from one nest ( $c$ is a photo of the nest with the grid overlaid). Distribution map drawn using a distance weighted least squares fit (with tension set to 0 ). The contours represent differing average weights of charcoal (in grams), and the open circle marks the nest entrance. 


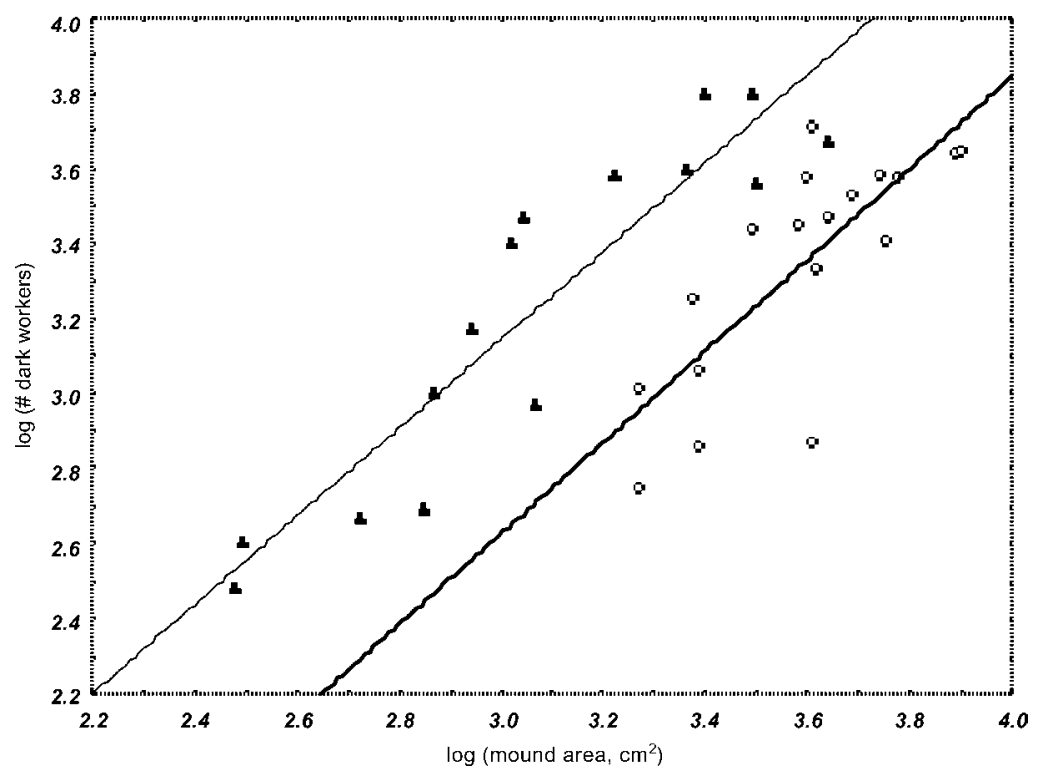

Fig. 2. Log-log relationship between mound area $\left(\mathrm{cm}^{2}\right)$ and colony size (\# dark workers). Open circles are colonies from CC 2003, closed triangles from AH 1989. The equation for $\mathrm{CC}$ is $y=-1.0223+1.2166 \times x$, and for $\mathrm{AH}$ is $y=-0.3918+1.1787 \times x$. At CC, $r^{2}=0.59$, and $p<0.001$, and at $\mathrm{AH}, r^{2}=0.84$, and $p<0.00001$.

Therefore, an increase in mound area yields a proportional increase in charcoal. The average weights of charcoal in the four collections were significantly different from each other after adjusting for colony size (Table I). At $\mathrm{AH}$, the colonies from across seasons in 1989 had the least charcoal, and in 2002 the amount of charcoal on nests doubled from May to October. At $\mathrm{CC}$ in May, the charcoal on nests was 4 times greater than the same season at AH. From this we conclude that the amount of charcoal on nests varies from year to year, across sites and seasons. The extremely low amount of charcoal in 1989 may be due to the greater elapsed time after a prescribed burn, and therefore lower availability of charcoal. Qualitative observations at sites throughout the ANF suggest that there is more charcoal on ant nests when there is more charcoal in the vicinity of colonies. Moreover, at a site burned the previous day foragers collected charcoal almost exclusively.

As colony size increases so does the amount of charcoal covering the nest. Colony size (\# of mature workers) accounts for $44 \%$ of the variance in the amount of charcoal atop the nest (AH). The slope of the relationship between colony size and charcoal is 0.81 , which is not significantly different 


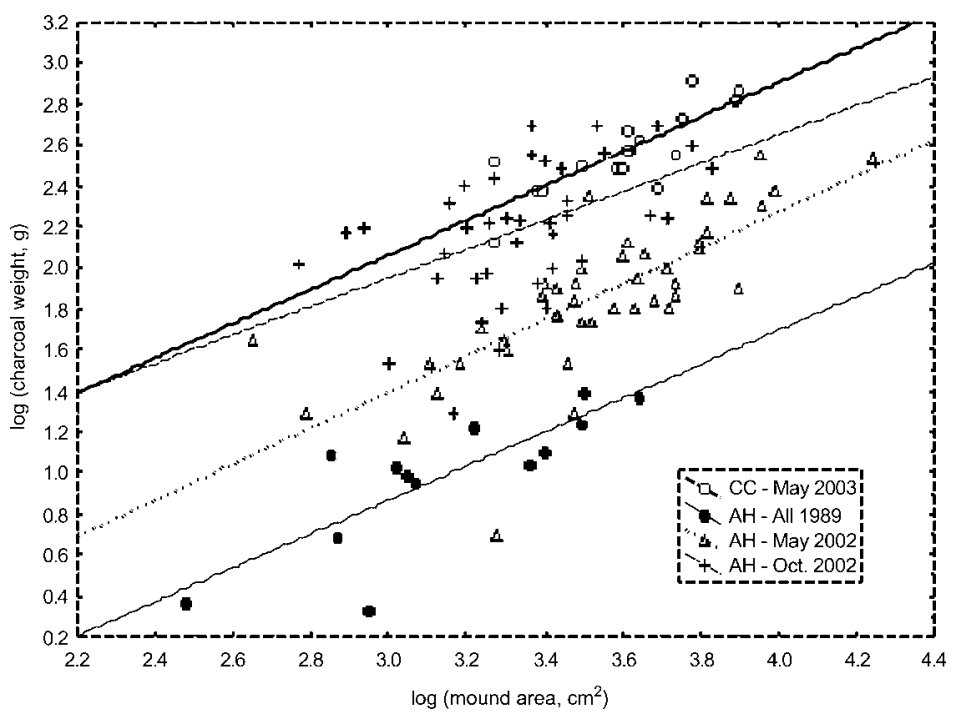

Fig. 3. Log-log relationship between mound area and charcoal weight for two sites, and several seasons. All slopes are not significantly different than 1, or isometry). The total weight of charcoal for any given colony size differed significantly for all four samples.

than 1 ( $t=0.93, d f=20, p>0.2$, isometry). Therefore it can be said that colonies of all sizes have similar amounts of charcoal per worker.

Method 1 of foraging estimation used a forager trap. The traps were opened (i.e., actively trapping) for an average of $35 \mathrm{~min}$ per trial ( 2 trials: $n=13$ colonies per trial). We eliminated one colony in the "charcoal removed" treatment because of an incomplete seal on the trap when trials were not active, which led to a halt in foraging activity. No statistical differences in collection rates (or even trends) were detected for any variable between treatment groups so we pooled data for further analysis.

A total of 984 foragers were examined over trials 1 and 2 (435 and 549 foragers in each trial, respectively). Approximately 1 forager was trapped per minute. The number of foragers trapped was proportional to the time the trap was open and did not diminish with time $[\log (\#$ foragers $)=-0.52$ $+1.31 \log ($ time $), r^{2}=0.33$; slope not different than $1: t=0.8$, df $=24$, $p>0.2]$. Thus, it does not seem likely that colonies adjusted their foraging rate due to negative feedback of missing foragers.

On average, three quarters of the foragers returned with a solid load. Proportions of foraged objects were not statistically different between trials and are pooled in the following calculations. Of the foraged objects, $42 \%$ 
Table II. Proportions of Foraged Items for three Species of Pogonomyrmex

\begin{tabular}{lccc}
\hline & \multicolumn{3}{c}{ Species } \\
\cline { 2 - 4 } & P. badius & P. barbatus & ${\text { P. } \text { salinus }^{a}}$ \\
\hline Nonfood objects & 0.29 & 0.35 & 0.22 \\
Seeds & 0.52 & 0.40 & 0.76 \\
Insects & 0.19 & 0.25 & 0.02 \\
\hline
\end{tabular}

${ }^{a}$ Data from Jorgensen and Porter (1982).

were unidentifiable (mostly portions of plant stems and leaves), $31 \%$ seeds $( \pm 17, \mathrm{SD}), 18 \%( \pm 16)$ charcoal, and $9 \%( \pm 9)$ insects (or parts thereof). Of the identifiable objects, $52 \%( \pm 28)$ were seeds, $29 \%( \pm 23)$ charcoal and 19\% $( \pm 26)$ insects (Table II). Unidentifiable objects are not included in our comparisons across species because it is likely that some of them fell into the collection cup independent of ant activity, and because they could not be definitively identified. Therefore, the proportion of nonfood objects we report is conservative by excluding nonfood objects other than charcoal. On average, colonies acquired 1 seed every $5 \mathrm{~min}$, a piece of charcoal every $8 \mathrm{~min}$, an insect every $20 \mathrm{~min}$, and other "stuff" every $3 \mathrm{~min}$.

Method 2 compared long-term charcoal accumulation atop nests for removal and control nests. This method ignores the collection of anything but charcoal. Colonies that had their charcoal removed collected $70 \%$ more charcoal than did colonies with intact depots (unweighted means \pm standard deviations: $128 \pm 49 \mathrm{~g}$ vs. $75 \pm 72 \mathrm{~g}$ respectively $)\left(F_{1,15}=17.8, p<0.001\right.$ after adjustment for colony size). Controls collected $23 \%$ of their starting weight. The percent gain of removal colonies could not be calculated because the treatments were initiated approximately 2 months prior to the beginning of charcoal quantification.

Novel objects, toothpick pieces, were only collected when presented with seeds. Of the eight colonies presented with objects and seeds, seven collected objects (observed result is different than random: $p<0.05$, binomial test). The one colony that did not retrieve objects stopped recruiting to the pile due to competition with recruiting foragers of Solenopsis geminata. Therefore, colonies did not recruit to objects alone despite foragers coming into contact with them. All colonies presented with seeds foraged all of the offered seeds.

Tests for object color and size preference do not appear to have been biased by changing ratios of color or size over time. Chi-square tests comparing the expected (2:1 for size and 1:1 for color) and observed ratios of 
uncollected objects yielded only one significant departure from expectation, there were more than expected uncollected green objects at a single colony.

Colonies placed an average of 382 of the offered objects on top of their nests. No colony showed a significant color preference (color nested within size: $F_{6,24}=0.07, p>0.9$ ). The lack of color preference negates the relevance of the above reported excess of green objects in one colony. All colonies showed significant preference for medium compared to large toothpick pieces (size: $F_{1,24}=95.2, p<0.0001$ ). Also of note is that all colonies retrieved all of the offered seeds, as in the above experiment.

In all observations on foragers returning to the nest with a toothpick piece, the piece was brought into the nest entrance $(n=24$ direct observations). In many cases, though, foragers dropped their objects between the pile of objects and the nest, or simply scattered the pieces immediately around the pile.

\section{P. barbatus}

The samples of pebbles from eight large $P$. barbatus colonies (nest area $\left.=22,165 \mathrm{~cm}^{2} \pm 7721\right)$ contained an average of 78 pebbles per $\mathrm{cm}^{2}$, indicating that the pebble covering was not superficial, but instead several centimeters deep. The weight of pebbles covering the nest was $41 \mathrm{~kg} \pm 26$, and the estimated number of pebbles was 1.7 million \pm 0.9 million. This estimate is likely quite liberal because it assumes that pebble density is the same over the entire mound, but the extent of the pebble covering is apparent even if this estimate is halved.

A total of 205 foragers were examined over the eight colonies. As in $P$. badius, no significant effect of treatment (pebbles removed vs. control) was detected for any variable. One half of the ants returned carrying no apparent load. On average 2.6 ants returned per minute with loads. Of these, $40 \%$ carried seeds (mostly from newly fallen Opuntia fruit), 35\% pebbles, and $25 \%$ insects (Table II). The rates of incoming seeds, pebbles and insects were approximately one per $1.25 \mathrm{~min}, 1.5 \mathrm{~min}$ and $5 \mathrm{~min}$ respectively.

\section{Comparative Data}

A review of the literature and personal communications with researchers (J. Cook, R. Johnson, W. Mackay, S. Porter, R. Snelling, and S. Taber) indicated that at least eight species of Pogonomyrmex collect nonfood objects, and deposit these objects atop their nests (Table III). An 
Table III. Comparison of Nonfood Depots of Pogonomyrmex Species

\begin{tabular}{lllll}
\multicolumn{1}{c}{$\begin{array}{c}\text { Species } \\
\text { name }\end{array}$} & $\begin{array}{c}\text { Species } \\
\text { complex }\end{array}$ & $\begin{array}{l}\text { Types of } \\
\text { objects }^{a}\end{array}$ & $\begin{array}{l}\text { Forage } \\
\text { objects? }\end{array}$ & $\begin{array}{c}\text { Information } \\
\text { source }^{b}\end{array}$ \\
\hline barbatus & barbatus & $\mathrm{P}, \mathrm{G}$ & yes & $\mathrm{CS}, \mathrm{RJ}, \mathrm{WM}, 1$ \\
rugosus & barbatus & $\mathrm{P}, \mathrm{G}$ & yes & $\mathrm{CS}, 1$ \\
tenuispina & barbatus & $\mathrm{P}$ & yes & $\mathrm{RJ}$ \\
wheeleri & barbatus & $\mathrm{P}$ & $?$ & $\mathrm{WM}$ \\
brevispinosus & occidentalis & $\mathrm{P}$ & $?$ & $\mathrm{RS}, 1$ \\
montanus & occidentalis & $\mathrm{P}$ & $?$ & $\mathrm{WM}$ \\
occidentalis & occidentalis & $\mathrm{P}, \mathrm{G}$ & yes & $\mathrm{RJ}, \mathrm{WM}, 1,2$ \\
salinus & occidentalis & $\mathrm{P}, \mathrm{G}$ & yes & $\mathrm{SP}$ \\
subdentatus & occidentalis & $\mathrm{P}$ & $?$ & 1 \\
subnitidus & occidentalis & $\mathrm{P}$ & $?$ & $\mathrm{WM}$ \\
badius & californicus & $\mathrm{C}, \mathrm{G}, \mathrm{D}$ & yes & $\mathrm{CS}$ \\
californicus & californicus & $\mathrm{D}$ & yes & 3 \\
maricopa & californicus & $\mathrm{P}, \mathrm{G}$ & yes & $\mathrm{RJ}, \mathrm{WM}, 1$ \\
\hline
\end{tabular}

${ }^{a}$ Types of objects, P: pebble, G: glass, C: charcoal, and D: debris.

${ }^{b}$ Information sources: letters represent personal communications or the author's observations $(\mathrm{CS}=$ Chris Smith, RJ $=$ Robert Johnson, RS = Roy Snelling, $\mathrm{SP}=$ Sanford Porter, and $\mathrm{WM}=$ William Mackay), and numbers represent published reports or pictures $(1=$ Cole, $1968 ; 2=$ Cole, $1932 ; 3=$ Baily and Polis, 1987).

additional five species have objects on their nests, but it is not known whether these objects derive from foraging or nest excavation.

There are many objects that occur on nests of several species. All of the western species surveyed have pebbles on their nests (Table III), and one with only plant debris. Glass fragments occur on the nests of $P . b a$ dius, barbatus, rugosus, occidentalis, maricopa, and salinus, especially along roadsides (personal observation).

The 13 species listed in Table III represent all three species-complexes, barbatus, occidentalis and californicus as described by Taber 1990 (but see Cole, 1968), and one half of the nonparasitic Pogonomyrmex of the USA and northern Mexico. Many of the remaining species live in sandy habitats that may or may not have objects to be collected and deposited on nests (e.g., no pebbles). For all of the remaining species there are no reliable behavioral observations on this behavior. In Taber's (1998) cladistic phylogeny of Pogonomyrmex, $P$. badius diverged very early in the history of the genus in N. America, indicating that this behavior is ancestral in the N. American species.

Table II shows the proportions of objects retrieved by foragers of P. badius, barbatus and salinus (=owyheei) (data on P. salinus are from Jorgensen and Porter, 1982). All of these species forage large quantities of nonfood objects of different types in similar relative amounts. 
Two Pogonomyrmex species are known occasionally to move nests (Gentry and Stiritz, 1972; Van Pelt, 1976; Harrison and Gentry, 1981), often in response to shading (Carlson and Gentry, 1973). P. barbatus moved their pebble depot when relocating their nest (VanPelt, 1976), and we have seen $P$. badius move part of their charcoal depot.

\section{DISCUSSION}

Our observations suggest that the ants may use overlapping cues when responding to food, charcoal and trash. The distributions of charcoal and midden (trash) on P. badius nests indicate that the manner in which the ants treat charcoal and midden are not independent (Fig. 1). This conclusion is strengthened by the observation of the ants taking offered toothpicks into the nest, and then placing them in their midden pile. This may be due to the same ants maintaining both the charcoal and midden piles (Gordon, 1984), where the "midden-workers" are more likely to deposit charcoal on midden piles, but the probability of dropping charcoal atop the pile is less than that for actual trash objects. Midden piles in some ants result from the increased probability of an ant dropping trash where trash is already dense (Howard and Tschinkel, 1976; Theraulaz et al., 2002). This positive feedback would explain why midden piles are typically discrete, while charcoal is more diffuse about the nest. Only rarely is charcoal found in the "granaries," seed storage chambers within the nest (personal observation). These observations support that the ant-charcoal relationship is not the same as the ant-trash or ant-food relationships, but there are likely overlapping cues governing how the ants respond to each of these items.

Seeing as the ants place more charcoal on areas containing trash it may be suggested that they use the charcoal as a "sanitizing filter" of sorts, binding up toxins that may be found in their trash. However, this charcoal is not activated and is unlikely to be effective in such a role. Furthermore, $P$. badius is the only species documented to have vast amounts of charcoal in its depot, and the only one that regularly encounters large quantities of charcoal. Most congeners live in sparse grassland or desert habitat where charcoal is normally absent.

Colonies grow by increasing the number and size of ants in the nest. The number of worker ants in a colony influences the reproductive/productive capacity in a $P$. badius colony (Tschinkel, 1999a,b), and therefore worker number is a good measure of colony size, or growth stage. Size is not necessarily tightly correlated with age in many species of ant (e.g., Tschinkel, 1993), making size a better estimate of the colony's developmental state (ontogeny). As colonies of P. badius increase in worker population, 
the size of their depots increases proportionately. This could simply be due to the forager population increasing as a function of the total worker population, where more nonfood objects are returned simply because more ants are foraging. This would imply that the proportion of nonfood objects foraged is constant. This appears true as the amount of charcoal on P. badius colonies changes isometrically with colony size, as do the number of seeds (Tschinkel, 1999a,b).

The proportions of foraged food objects brought back to the nest by $P$. badius differed from a previous study (Traniello and Beshers, 1991), where only a small proportion $(6 \%)$ of foragers returned with nonfood objects (or at least "unidentifiable plant parts"), and none were observed returning with charcoal, glass or other typical objects found on P. badius nests. That study also reported a higher intake of insects $(43 \% \pm 35)$ than our data $(19 \% \pm 26)$; these differences are likely due to population and habitat differences, a larger sample size of colonies in our study (13 vs. 2 colonies), and a high variability of foraged objects.

Although short-term data on P. badius and P. barbatus foragers indicates that colonies retrieve equal amounts of nonfood objects regardless of whether their depots were removed, long-term data on P. badius demonstrate that the colonies lacking charcoal piles increased their retrieval rate of charcoal. This discrepancy is likely due to timing because foraging traps were opened on days preceding treatment maintenance (charcoal removal or removal and replacement). Therefore, colonies lacking charcoal depots may have increased their rate of foraging on charcoal immediately after removal, but slowly lessened this rate over time as they accumulated more charcoal. The increase in collection rate could be due to either an increase in total foraging or a selective increase in foraging for charcoal. If the seed stores in the colonies reflect their foraging effort for seeds, the colonies in both treatments did not differ (unpublished data). Therefore, it appears likely that colonies actively increased their rate of foraging for charcoal when they were deprived of their depots. Gordon (1984) obtained a similar result, colonies deprived of their charcoal increased foraging rates for charcoal, but overall food foraging rates did not change. Furthermore, O'Donnell and Jeanne (1990) report a similar result for the wasp, Polybia occidentalis, where after nest damage foraging for nest materials increased, but not at the expense of food foraging. The absence of charcoal on nests, or damage to nests, stimulates colonies to forage nonfood objects at a higher than usual rate.

Colonies with their depots removed did not retrieve objects when they were offered alone, but did when in the presence of seeds. This result indicates that object retrieval is context-dependent, at least when foragers are recruited to areas. Colonies do exercise preference in the objects that they 
collect, preferring medium over large toothpick pieces, but not choosing based on color, or a combination of color and size.

Nonfood object collection appears to be an ancestral, and possibly homologous, behavior in the North American Pogonomyrmex. Not only do many species collect similar objects, but they also appear to collect the objects at high rates (or in seemingly similar proportions, Table II). As each object retrieved is the result of one foraging trip by one worker, each nonfood object represents the opportunity cost of a food item not returned, and includes the costs involved in retrieval (time, energy, and mortality). Estimates of the rate of nonfood foraging for $P$. badius and $P$. barbatus, along with the number of objects atop their nests, the movement of piles following nest relocation, and the effort involved in maintaining the piles suggest that this is a costly behavior. Interestingly, foraging in the genus is not thought to be energetically costly as the benefit-to-cost ratio of a forager returning with a seed is greater than 1000 (Fewell, 1988; Weier et al., 1995). Even though a large number of foragers return with nonfood or no load (or die while foraging), the high energetic benefit derived from seeds is likely to offset these energetic costs. Moreover, studies of the bioenergetics of harvester ants estimate that colonies are not energy limited (i.e., energy intake exceeds use) (MacKay, 1985; Golley and Gentry, 1964, but see Porter, 1986), and seasonally unchanging seed caches in $P$. badius support this notion (Tschinkel, 1999a,b). Therefore, if the behavior is costly, it is likely so through time (Weier and Feener, 1995; Fewell et al., 1996) and opportunity costs. Through time, the behavior increases in cost proportionately to colony size, and through opportunity (also in units of time) at the expense of doing an alternate task (e.g., food collection).

Feedback of the depot on colony worker allocation is evidenced by depot removal stimulating its active recollection through increased worker allocation into this task, but not increased food foraging. Recruitment to objects is dependent on the presence of food, and the behavior of colonies toward food, objects and trash overlap. Taken together, this suggests that depot formation is the result of the integration of many tasks, all of which alone are important in colony function. Therefore, selection on depot formation occurs indirectly through selection on various colony-level optimization pathways (e.g., food collection, food processing and trash elimination). Selection for increased worker variability and flexibility in their response to cues is a possible mechanism for increased efficiency in total task allocation, but may allow for the evolution of new tasks and extended phenotypes (e.g., object collection and depot formation) (Dawkins, 1976).

Though we believe the information we report is compelling evidence for the generalization of this behavior across the genus, several limitations must be addressed. The lack of information on many species, along with 
the lack of a well resolved phylogeny, limit any conclusions that we can make about the homology of the behavior in the genus. Moreover, asserting that the ontogeny of the behavior is similar across species is complicated by the difficulty of excavating a sufficient sample of colonies in most western species (rocky soil). And though our conclusions on the stimulation of nonfood collection agree with those of Gordon (1984), there is no information to support the generalization of this stimulus in other species.

The collection of nonfood objects and the sometimes-colossal depots that are formed with their deposition at a central place is not only a mysterious behavior, but understanding it can give insights into many other topics such as optimal foraging, search images, and the influence of nest superstructure on colony organization, physiology, and fitness. Harvester ants have been used in many studies of optimal foraging (e.g., Baily and Polis, 1987; Fewell, 1988; Weier et al., 1995; Morehead and Feener, 1998), but these studies neglect the retrieval of nonfood objects. Given that nonfood foraging occurs at such a high rate in some harvester ants, its inclusion in optimal foraging thinking will aid in our understanding of the "optimality" of foraging. Optimality in all cases should be viewed as relative to the optimization of the whole colony, as this is manifested through many dependent pathways. Only through an integrated approach can we understand how emergent properties arise in social systems, as they are always the result of selection on varying levels and through varying pathways.

\section{ACKNOWLEDGMENTS}

The authors are grateful to E. Burke, who helped process samples in the lab, A. Winn and B. Inouye for comments and help with the manuscript. K. Haight, A. Mikheyev, J. Seal and S. Storz, all mentally and physically contributed to these projects. This manuscript was in partial fulfillment of a MS degree at Florida State University for CRS. Funding was provided by the Short Fellowship (CRS) and the FSU Dept. of Biological Science. All experiments complied with the laws of the United States of America.

\section{REFERENCES}

Baily, K. H., and Polis, G. A. (1987). Optimal and central-place foraging theory applied to a desert harvester ant, Pogonomyrmex californicus. Oecologia 72: 440-448.

Carlson, D. M., and Gentry, J. B. (1973). Effects of shading on the migratory behavior of the Florida harvester ant, Pogonomyrmex badius. Ecology 54: 452-453.

Cole, A. C., Jr. (1932). The relation of the ant, Pogonomyrmex occidentalis Cr., to its habitat. Ohio J. Sci. 32: 133-146. 
Cole, A. C., Jr. (1968). Pogonomyrmex Harvester Ants. A Study of the Genus in NORTH America, University of Tennessee Press, Knoxville, TN.

Dawkins, R. (1976). The Selfish Gene, Oxford University Press, Oxford, UK.

Fewell, J. H. (1988). Energetic and time costs of foraging in harvester ants, Pogonomyrmex occidentalis. Behav. Ecol. Sociobiol. 22: 401-408.

Fewell, J. H., Harrison, J. F., Lighton, J. R. B., and Breed, M. D. (1996). Foraging energetics of the ant, Paraponera clavata. Oecologia 105: 419-427.

Gentry, J. B., and Stiritz, K. L. (1972). The role of the Florida harvester ant, Pogonomyrmex badius in old field mineral nutrient relationships. Environ. Entomol. 1: 39-41.

Golley, F. B., and Gentry, J. B. (1964). Bioenergetics of the southern harvester ant, Pogonomyrmex badius. Ecology 45: 217-225.

Gordon, D. M. (1984). The harvester ant (Pogonomyrmex badius) midden: Refuse or boundary? Ecol. Entomol. 9: 403-412.

Gordon, D. M. (1999). Ants at Work: How an Insect Society is Organized, Free Press, New York.

Gould, S. J., and Lewontin, R. C. (1979). The spandrels of San Marco and the Panglossian paradigm: A critique of the adaptationist programme. Proc. R. Soc. Lond. B 205: 581598.

Harrison, J. S., and Gentry, J. B. (1981). Foraging pattern, colony distribution, and foraging range of the Florida harvester ant, Pogonomyrmex badius. Ecology 62: 1467-1473.

Hatcher, J. B. (1896). Some locations for Laramie mammals and horned dinosaurs. Am. Nat. 30: $112-120$.

Howard, D. F., and Tschinkel, W. R. (1976). Aspects of necrophoric behavior in the red imported fire ant, Solenopsis Invicta. Behav. 56: 157-180.

Jorgensen, C. D., and Porter, S. D. (1982). Foraging behavior of Pogonomyrmex owyheei in southeast Idaho. Environ. Entomol. 11: 381-384.

Laundre, J. W. (1990). Soil moisture patterns below mounds of harvester ants. J. Range Manage. 43: $10-12$.

Lull, R. S. (1915). Ant-mound fossils. Pop. Sci. Mon. 87: 236-243.

MacKay, W. P. (1985). A comparison of the energy budgets of three species of Pogonomyrmex harvester ants (Hymenoptera: Formicidae). Oecologia 66: 484-494.

MacKay, W. P., and MacKay, E. E. (1985). Temperature modifications of the nest of Pogonomyrmex montanus (Hymenoptera: Formicidae). Southwest. Nat. 30: 307-309.

Morehead, S. A., and Feener, D. H., Jr. (1998). Foraging behavior and morphology: Seed selection in the harvester ant genus, Pogonomyrmex. Oecologia 114: 548-555.

O'Donnell, S., and Jeanne, R. L. (1990). Forager specialization and the control of nest repair in Polybia occidentalis Olivier (Hymenoptera: Vespidae). Behav. Ecol. Sociobiol. 27: 359364.

Porter, S. D. (1986). Revised respiration rates for the southern harvester ant, Pogonomyrmex badius. Comp. Biochem. Physiol. A Comp. Physiol. 83: 197-198.

Reynolds, T. D. (1991). Movement of gravel by the "Owyhee" harvester ant, Pogonomyrmex salinus (Hymenoptera: Formicidae). Entomol. News 102: 118-124.

Sober, E.,493 and Wilson, D. S. (1998). Unto Others: The Evolution and Psychology of Unselfish Behavior, Harvard University Press, Boston.

Smith, C. R. (2004). Florida harvester ants and their charcoal. Masters Thesis, Florida State University, Tallahassee, FL.

Taber, S. W. (1998). The World of the Harvester Ants, Texas A\&M University Press Natural History Series, College Station, TX.

Theraulaz, G., Bonabeau, E., Nicolis, S. C., Sole, R. V., Fourcassie, V., Blanco, S., Fournier, R., Joly, J.-L., Fernandez, P., Griomal, A., Dalle, P., and Deneubourg, L-L. (2002). Spatial patterns in ant colonies. Proc. Natl. Acad. Sci. U.S.A. 99: 9645-9649.

Tinbergen, N. (1963). On the aims and methods of ethology. Z. Tierpsychol. 20: 410-433.

Traniello, J. F. A., and Beshers, S. N. (1991). Polymorphism and size-pairing in the harvester ant Pogonomyrmex badius: A test of the ecological release hypothesis. Insectes Soc. 38: 121-127. 
Tschinkel, W. R. (1993). Sociometry and sociogenesis of colonies of the fire ant Solenopsis invicta during one annual cycle. Ecol. Mongr. 64: 425-457.

Tschinkel, W. R. (1998). Sociometry and sociogenesis of colonies of the harvester ant, Pogonomyrmex badius: Worker characteristics in relation to colony size and season. Insectes Soc. 45: 385-410.

Tschinkel, W. R. (1999a). Sociometry and sociogenesis of colony-level attributes of the Florida harvester ant (Hymenoptera: Formicidae). Ann. Entomol. Soc. Am. 92: 80-89.

Tschinkel, W. R. (1999b). Sociometry and sociogenesis of colonies of the harvester ant, Pogonomyrmex badius: Distribution of workers, brood and seeds within the nest in relation to colony size and season. Ecol. Entomol. 24: 222-237.

Tschinkel, W. R. (2004). Nest architecture of the ant, Pogonomyrmex badius. J. Insect Sci. 4: 21.

Van Pelt, A. F. (1976). Nest relocation in the ant Pogonomyrmex barbatus. Ann. Entomol. Soc. Am. 69: 493.

Weier, J. A., and Feener, D. H., Jr. (1995). Foraging in the seed-harvester ant genus Pogonomyrmex: Are energy costs important? Behav. Ecol. Sociobiol. 36: 291-300.

Weier, J. A., Feener, D. H., Jr., and Lighton, J. R. B. (1995). Inter-individual variation in energy cost of running and loading in the seed-harvester ant, Pogonomyrmex maricopa. J. Insect Physiol. 41: 321-327.

Whitford, W. G. (2003). The functional significance of cemented nest caps of the harvester ant, Pogonomyrmex maricopa. J. Arid Environ. 53: 281-284. 